\title{
XBP1-US/XBP1-SP/CD138/CS1 \\ Multipeptide Vaccine PVX-410
}

National Cancer Institute

\section{Source}

National Cancer Institute. XBP1-US/XBP1-SP/CD138/CS1 Multipeptide Vaccine PVX-410.

NCI Thesaurus. Code C103823.

A cancer vaccine containing immunog enic, HLA-A2-specific epitopes derived from X-boxbinding protein 1-unspliced (XBP1-US), XBP1-spliced (SP), syndecan-1 (CD138), and CS1 (CD2 subset 1, CRACC, SLAMF7, CD319) with potential immunomodulating and antineoplastic activities. Upon subcutaneous administration, XBP1-US/XBP1SP/CD138/CS1 multipeptide vaccine PVX-410 may stimulate the immune system to induce a cytotoxic T-lymphocyte response against the four myeloma-specific antigens. The tumor associated antigens (TAAs) XBP1-US, XBP1-SP, CD138 and CS1, are overexpressed on the surface of multiple myeloma (MM) cells. 